\title{
Selective Self-Adaptive Approach to Ant System for Solving Unit Commitment Problem
}

\author{
${ }^{1}$ Songsak Chusanapiputt $\quad{ }^{2,3}$ Dulyatat Nualhong ${ }^{3}$ Sujate Jantarang ${ }^{4}$ Sukumvit Phoomvuthisarn \\ ${ }^{1}$ Dept. of Electrical Power Eng. \\ Faculty of Engineering \\ Mahanakorn University of Tech. \\ Bangkok, Thailand \\ ${ }^{2}$ Electrical Eng. Dept. $\quad{ }^{3}$ Dept. of Computer Eng. \\ Elect. \& Control Sys. Division Faculty of Engineering \\ ${ }^{4}$ Center of Excell. in Elect. Power Tech. \\ EGAT \\ Nonthaburi, Thailand \\ Mahanakorn University of Tech. \\ Bangkok, Thailand \\ E-mail: songsak@mut.ac.th E-mail: dulyatat.n@egat.co.th \\ E-mail: sujate@mut.ac.th \\ Faculty of Engineering \\ Chulalongkorn University \\ Bangkok, Thailand \\ E-mail: sukumvit.p@chula.ac.th
}

\begin{abstract}
This paper presents a novel approach to solve the constrained unit commitment problem using Selective Self-Adaptive Ant System (SSAS) for improving search performance by automatically adapting ant populations and their transition probability parameters, which cooperates with Candidate Path Management Module (CPMM) and Effective Repairing Heuristic Module (ERHM) in reducing search space and recovering a feasible optimality region so that a high quality solution can be acquired in a very early iterative. The proposed SSAS algorithm not only enhances the convergence of search process, but also provides a suitable number of the population sharing which conducts a good guidance for trading-off between the importance of the visibility and the pheromone trail intensity. The proposed method has been performed on a test system up to 100 generating units with a scheduling time horizon of 24 hours. The numerical results show the most economical saving in the total operating cost when compared to the previous literature results. Moreover, the proposed SSAS topology can remarkably speed up the computation time of ant system algorithms, which is favorable for a large-scale unit commitment problem implementation.
\end{abstract}

\section{Categories and Subject Descriptors:}

I.2.1 [Artificial Intelligence]: Application and Expert SystemsIndustrial Automation; I.2.8 [Artificial Intelligence]: Problem Solving, Control Methods, and Serach- heuristic methods

General terms: Algorithms, Performance, Experimentation

Keywords:

Unit commitment, Ant system, Selective Self-Adaptive approach

\section{INTRODUCTION}

Unit Commitment (UC) is one of the most important plans for the electric utility company to determine most economical start-up and shut-down times for the electrical power generating units to meet a load demand while satisfying a set of operational constraints. As the energy market moves toward deregulation, the UC schedule directly affects the production cost, making it an essential input to successful bidding strategy. It is recognized that UC problem is a large and complex mathematical optimization with both integer and continuous variables. The exact solution to the problem can be obtained only by

Permission to make digital or hard copies of all or part of this work for personal or classroom use is granted without fee provided that copies are not made or distributed for profit or commercial advantage and that copies bear this notice and the full citation on the first page. To copy otherwise, or republish, to post on servers or to redistribute to lists, requires prior specific permission and/or a fee.

GECCO'06, July 8-12, 2006, Seattle, Washington, USA.

Copyright 2006 ACM 1-59593-186-4/06/0007...\$5.00. complete enumeration, which cannot be applied to a realistic power system due to its excessive computation time requirement [1], [2].

The Lagrangian Relaxation (LR) method is a fair agreement between solution quality and computation requirement for a largescale UC problem [3], [4]. Although LR method can provide a fast solution but sometime it suffers from numerical convergence problem especially when the problem is non-convex. Beside, this method strongly depends on the technique used to update Lagrange multipliers. Most researchers dealing with LR use gradient method to achieve this task. However, the solution obtained from gradient-based method undergoes from convergence problem and always gets stuck into a local optimum.

In order to overcome these problems of UC, many stochastic optimizations such as Genetic Algorithms (GA) [5] and Evolutionary Programming (EP) [6] were introduced into power system optimization. These methods begin with a population of starting points, and use only the objective function information for searching. Therefore, these methods can provide a high-quality solution. However, GA and EP always suffer from a big problem, that they take a long computational time of evolving process.

Recently, Ant System (AS) [7] is a class of heuristic search algorithms that is inspired by the behavior of colonies of real ants, and in particular how they forage for food, and have been successfully applied to Traveling Salesman Problem with better solution than GA in performance. In the literatures of [8] and [9], many AS approaches have been applied to combinatorial optimization problems of UC planning with good results. Nevertheless, AS still strongly needs many techniques to develop it to be better when applied to large-scale UC problem solving. Therefore, this paper employs ant system algorithms based on the selective self-adaptive approach combined with many newly developed techniques for providing a beneficial attribution to AS application in the field of UC problem, and improving the performance of AS algorithms in such the way of highquality UC solution within a reasonable computation time for solving a large-scale UC problem.

\section{PROBLEM FORMULATION}

\subsection{Notation}

$i \quad$ Index of generating unit;

$t \quad$ Index of unit commitment hour;

$k \quad$ Index of ant agent based ranking;

$u \quad$ Index of searching iteration;

$j \quad$ Index of candidate path;

$a_{i}, b_{i}, c_{i} \quad$ Cost function parameters of unit $i$;

$P_{i, t} \quad$ Generation of unit $i$ at hour $t$;

$F_{i, t}\left(P_{i, t}\right) \quad$ Fuel cost of unit $i$ at hour $t(\$ / \mathrm{h})$;

FT Total operating cost over the scheduling horizon (\$); 
$P \min _{i} \quad$ Unit $i$ minimum generation limit (MW);

$P \max _{i} \quad$ Unit $i$ maximum generation limit (MW);

$D_{t} \quad$ System peak demand at hour $t$ (MW);

$R_{t} \quad$ System reserve at hour $t(\mathrm{MW})$;

$S C_{i, t} \quad$ Start-up cost of unit $i$ at hour $t$;

$T \quad$ Scheduling time horizon;

$N \quad$ Number of generating unit;

Tup $_{i} \quad$ Unit $i$ minimum up time;

Tdown $_{i} \quad$ Unit $i$ minimum down time;

$X_{i, t} \quad$ Unit $i$ status at hour $t:=1$ if the unit is ON and 0 if OFF at hour $t$;

$V_{i, t} \quad$ Unit $i$ start-up/shutdown status at hour $t:=1$ if the unit is started at hour $t$ and 0 otherwise;

Tcold $_{i} \quad$ Cold start hour of unit $i$;

$\operatorname{Xoff}_{i} \quad$ Duration during which unit $i$ is continuously OFF;

$\mathrm{Xon}_{i} \quad$ Duration during which unit $i$ is continuously ON;

$H S T_{i} \quad$ Hot start-up cost of unit $i$;

$\mathrm{CST}_{i} \quad$ Cold start-up cost of unit $i$.

\subsection{Objective function}

The major cost of thermal generating units is the fuel cost of the committed units that is conventionally taken in a quadratic form.

$$
F_{i, t}\left(P_{i, t}\right)=a_{i} P_{i, t}^{2}+b_{i} P_{i, t}+c_{i}
$$

\$hour

Accordingly, the overall objective function of the constrained UC problem by the total operating cost is:

$$
\min F T=\sum_{t=1}^{T} \sum_{i=1}^{N}\left(X_{i, t} F_{i, t}\left(P_{i, t}\right)+V_{i, t} S C_{i, t}\right) \quad \$
$$

\subsection{Constraints}

\subsubsection{Load demand:}

All committed units must satisfy the power demand in each forecasted UC hour.

$$
\sum_{i=1}^{N} X_{i, t} P_{i, t}=D_{t} \quad 1 \leq t \leq T
$$

\subsubsection{Spinning reserve}

Spinning reserve is the total amount of generation capacity available from all units synchronized (spinning) on the system minus the present load demand. This is necessary due to certain outage of equipment. Spinning reserve requirement may be specified in terms of excess generation capacity or some form of reliability measure.

$$
\sum_{i=1}^{N} X_{i, t} P \max _{i} \geq\left(D_{t}+R_{t}\right) \quad 1 \leq t \leq T
$$

\subsubsection{Generation limits}

Generation limits ensure that the generated power provides the minimum and maximum capacity requirements of the corresponding generators for all UC hours.

$$
X_{i, t} P \min _{i} \leq P_{i, t} \leq P \max _{i} X_{i, t} \quad 1<t \leq T, \quad 1<i \leq N_{(5)}
$$

\subsubsection{Minimum up/down time}

Due to the characteristics of generators, the minimum uptime and downtime constraint assures that a generator will stay at the state for at least a given length of time.

$$
\left.\begin{array}{l}
\operatorname{Xoff}_{i} \geq \text { Tdown }_{i} \\
\text {Xon }_{i} \geq \text { Tup }_{i}
\end{array}\right\}
$$

In this work, the start-up cost depends upon the types of start-up method: Hot Start-up (HST) or Cold Start-up (CST), in order to calculate the start-up cost characteristics of each generator.

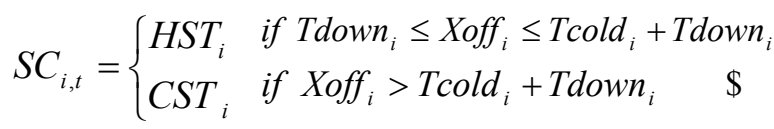

\section{THE PROPOSED METHODOLOGY}

Ant System (AS) has been developed by Dorigo et al. [10], [11]. It is proposed as a viable new approach to stochastic combinatorial optimization. The idea is inspired by an observation of real ant behaviors. A colony of ants is able to succeed in a task to find the shortest path between the nest and the food source. It was found that ants deposit a chemical substance trail, called pheromone, on the ground when they move. This pheromone can be observed by other ants and motivates them to follow the path with a high probability. That is how the trail is reinforced and many ants follow that trail. The final result is that very soon all ants will choose the shortest path in their movement.

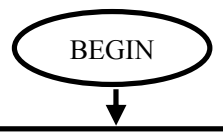

Block 1: Initialize parameters and search space

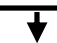

Block 2: Candidate Path Management Module (CPMM)

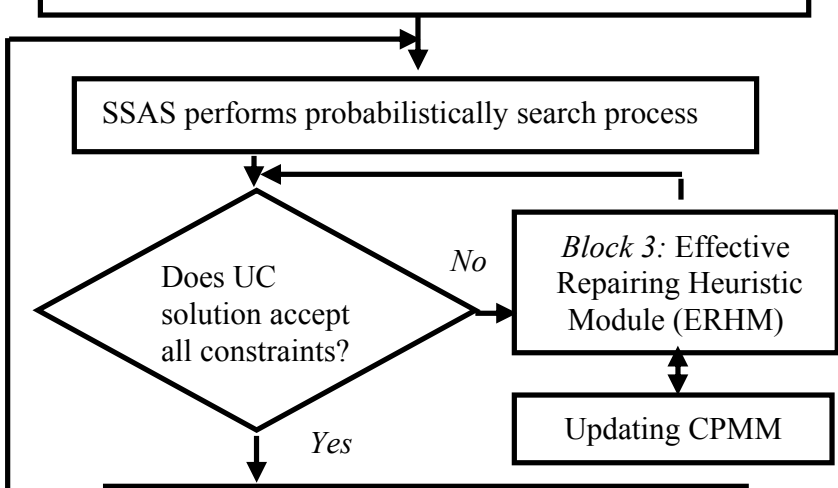

Block 4: Selective Self-Adaptive Approach

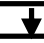

Pheromone Updating based Diversity Control Approach

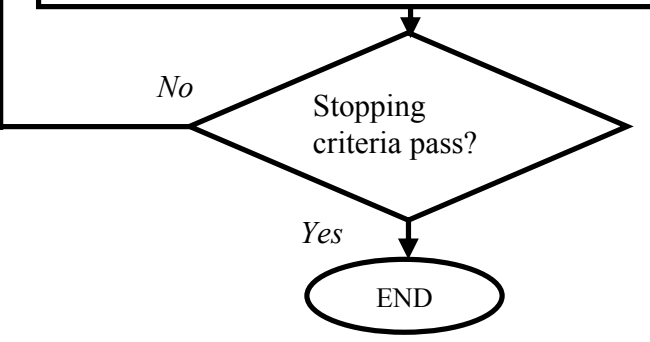

Figure 1. Overall flowchart of the proposed SSAS topology

Fig. 1 shows the flowchart of the proposed SSAS topology. The 
details of the proposed method are described below. There are four essential blocks, which represent the overall idea.

\subsection{Block 1: Initialize parameters and search space}

For the initialization of the proposed algorithm, the input data of generators including forecasted load demand are assigned to the SSAS program. The representation of UC solution is important. In our solution, the bit strings consist of the status ON/OFF for each generating unit during the UC hour horizon represented as ' 1 ' or ' 0 ', respectively. The length of the bit strings in the columns is equal to the number of generating units. This paper is to consider a future 24hour of UC problem so that 24 bits strings are given for the rows of UC solution format. Then, the initial parameters of pheromone trail intensity, transition probability, and the initial of ant populations are also declared at this step. By the proposed SSAS, the number of ant populations can be adaptively changed during the search process according to the proposed selective self-adaptive approach.

$$
A I M P_{i}=\frac{\left(d F_{i, t} / d P_{i, t}\right)_{P \max _{i}}}{P_{\max _{i}}}
$$

Here, a new priority index in (8), named as the Average Increment Maximum Power (AIMP), is created based on each generating unit's characteristics, and is suggested to be used along with the proposed SSAS approach. Respecting to AIMP index in sorted ascending, the status of each generator is set to ' $\mathrm{ON}$ ' until the total generation is sufficient for the spinning reserve constraint in (4) in every UC hour.

\subsection{Block 2: Candidate path management module}

As we know, the nature of UC problems contains many combination search spaces that rise up exponentially when the number of generating units is increased. To create a multi-stage search space, the Block 2 of the Candidate Path Management Module (CPMM) is introduced as a tool to propagate only the essential combinations from big search space, as much as possible to diminish UC search domain, which is an improved way of reducing computational time burden. This CPMM also records information such as the economic dispatch solutions and SSAS searching history while the program is running. Giving to the CPMM candidate paths, the UC bit string combinations for each hour are trimly selected within the range of $1.1 D_{t}$ to $1.5 D_{t}$ based on the AIMP index. While running SSAS program, the CPMM can be automatically updated depending on the new comings of candidate paths, which are generated by the proposed effective repairing heuristic module. This set of feasible candidates is supposed never to be empty and they are tested for the solution satisfaction of the forecasted load demand according to the spinning reserve in (4) against the upper and lower bound of total generating capacity in (5). This proposed method does not only propagate as many as possible ways to cover search regions, but also reduces CPU memory by collecting only the essential candidate paths. Consequently, the optimal UC solution can be acquired at a very early stage.

\subsection{Block 3: Effective repairing heuristic module}

For Block 3, the process of repairing or correcting the violated schedule is addressed by means of a new approach, named as the Effective Repairing Heuristic Module (ERHM), which is the heuristic defined to be used for recovering an infeasible UC solution for the satisfactions of certain performances criteria, and to produce a highquality feasible UC solution. In this paper, many new heuristics are introduced to satisfy the UC constraints in the following:

\subsubsection{The ON/OFF majority classification heuristic}

From the UC solution after ant agents generate their tour along UC hours planning, the $\mathrm{ON} / \mathrm{OFF}$ status of generating units is already arranged from the data base of CPMM. Next, each generating unit is classified into "ON-majority" and "Off-majority". Horizontally, if the statuses of whatever unit are ' 0 ' more than 12 hours, it is set to be as "Off-majority", and then the unit statuses between the first hour to the last hour which show ' 0 ' in that unit will be set to be ' 0 '. On the contrary, if the statuses of whatever unit are ' 1 ' more than 12 hours, it is set as "ON-majority", and then, the unit statuses between the first hour and the last hour which show ' 1 ' will be set to be ' 1 ' as well. Generally speaking, this heuristic tends to modify the ON/OFF unit status to be the continuous operation on the majority way that unit is operating for saving the total start-up cost of UC planning.

\subsubsection{Early Starting-up heuristic}

Due to the hot start-up having a cheaper cost than the cold start-up, this heuristic attempts to operate generating units continuously and start them up as fast as possible within the hot-starting hour in respect to the statuses of generating units for saving the total start-up cost. Considering UC hours, if the cost of early starting-up is lower when compared to the economic dispatch cost by a well-know $\lambda$-iteration method [1], this heuristic changes the unit statuses form ' 0 ' to ' 1 ' horizontally, and then moves to do the same on the next upper units ordering to the proposed AMIP index. Conversely, if there is no improvement, it will stop and go back to the prior solution.

\subsubsection{Stamping heuristic based Probabilistic Start-up Index}

Because of some UC hours appearing insufficiently below the load demand constraint in (3) or the spinning reserve constraint in (4), these hours need to start up other units to provide enough generation power. Here, the classification is revised again; the units are classified into the two types, that are "Free start-up unit" (FS) and "Non-free start-up unit" (NFS). The FS is the unit which has minimum up time equal to 1 hour. The NFS is the unit which has minimum up time more than 1 hour. As shown in Fig. 2 for a 10-unit system, "Sufficient hour" is for the unaccepted hour of the spinning reserve constraint in (4), and "Insufficient hour" for the accepted hour of the spinning reserve constraint in (4).

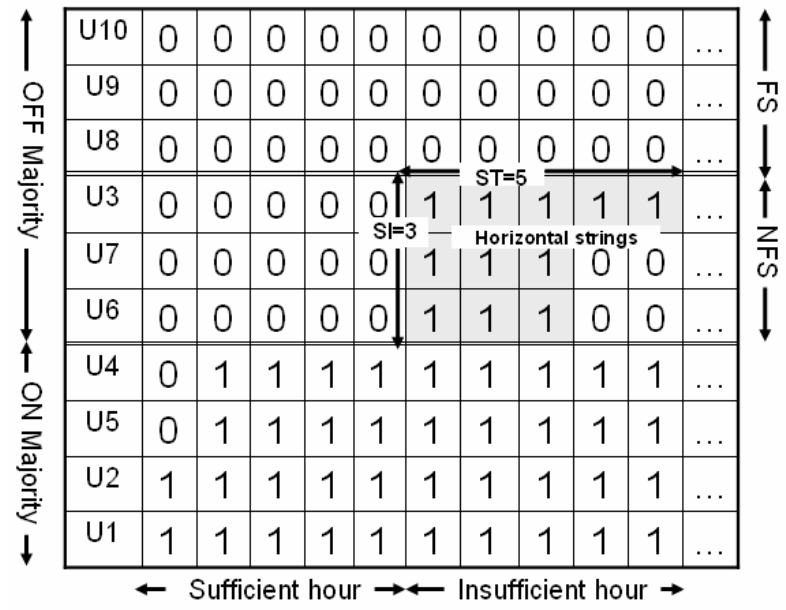

Figure 2. Illustration of the proposed stamping heuristic

This new heuristic probabilistically decides to stamp "Horizontal strings", beginning since the first "Insufficient hour" to the last on the 
classified "Off-majority" units of NFS. On this way of the approach, "Horizontal string", is set up specifically filled with ' 1 ', and its length is equal to minimum up-time of the considered unit. If "Horizontal string" is longer than the last UC hour, then, it is cut in range of the last hour UC planning.

The first column of Fig. 2 is generating unit ranking based on the AIMP index. Especially for NFS zone, this heuristic is designed to rank them according to their start-up cost, as shown in Fig.2, comparing among U3, U7 and U6, the cheapest unit (U6) is located on the bottom. In cases of a large-scale UC problem, there is often a large number of generating units. To overcome this problem, the number of units which are to be stamped is in range of $1 / 2$ to $3 / 4$ of the NFS zone. The number of stamping units can be determined probabilistically by proposing Useful Power Index (UPI) and the Probabilistic Start-up Index (PSI) as follows:

$$
\begin{aligned}
& U P I_{S I}=\sum_{s t=1}^{S T}\left|\left(\sum_{s i=1}^{S I} P_{s i, s t}\right)-D_{s t}+R_{s t}\right| \\
& P S I_{S I}=\frac{\left(1 / U P I_{S I}\right)}{\sum_{S I=1}^{S I^{\max }}\left(1 / U P I_{S I}\right)}
\end{aligned}
$$

where $s i$ is the index of the unit in range of NFS, when $s i=(1,2, \ldots, S I)$, $S I$ is the index of the last unit in range of NFS, when $S I=\left(1,2, \ldots, S I^{\max }\right), S I^{\max }$ is the index of the maximum last unit in range of NFS, st is the index of the hour in range of "Horizontal strings", when $s t=(1,2 \ldots, S T)$, and $S T=$ the longest string in the group of horizontal strings

In the proposed method, the PSI reflexes a possible chance to have a good stamping position in the number of generating units of NFS, referring to the usefulness of all gained generation power based UPI against the spinning reserve constraint in (4) for every hour located in length of "Horizontal strings". Based on a well-known Roulette Wheel selection process, this PSI provides diversity behavior to the candidate paths of CPMM automatically so that there is no worry that SSAS will be stuck on a premature convergence solution. After that, the sum of generation power provided by the stamping heuristic for the combinations is near to satisfying the spinning reserve constraint in (4), the probability of the combinations being optimum is high, but some hours may be over or below the constraint in (4). Excluding NFS zone, for an excess generation hour, this heuristic looks to change ' 1 ' to ' 0 ' status according to the AIMP index until it meets the constraint in (4). Inversely, for a lacking generation hour, this heuristic assigns to change ' 0 ' to ' 1 ' status in the same fashion with no violation of the minimum up-down time constraint in (6). For a large number of units, the ERHM may need more than one time of heuristics process to fix them, but the final solution is promisingly observed to be feasible in all improvements.

\subsection{Block 4: Selective self-adaptive approach}

It has been noted in the literature that the initial ant population and transition probability parameters can play a role in the performance of AS algorithms [7] so that some techniques should be invented for the setting of these parameters, otherwise we must take time to find the optimal constants or it may converge to the local minimum. This proposed method improves the performance of AS algorithms by automatically adjusting the number of ant populations and transition probability parameters with a significant margin comparing to the recording information and solutions in the neighborhoods of CPMM. The process of selective self-adaptive approach is updated every iteration based on a new concept of population sharing for the UC solution evaluation as described in section 4 .
After completing Block 4, SSAS moves to do pheromone updating based the diversity control approach [12], and then, the process will end if "stopping criterion" is satisfied, otherwise it is back to continue the search process. There may be several possible conditions to stop the search. In this paper, we use the counter of cycles performed since the best solution last changed is greater than a setting value.

\section{SELECTIVE SELF-ADAPTIVE APPROACH}

In our context of the proposed selective self-adaptive approach, the objective of this development is to reinforce search performance for ambiguous UC solution hours. The number of ant agents can be selectively transferred, and their transition probability parameters can also be adjusted for good trading-off between the visibility and the pheromone trail intensity of each UC hour planning. Generally, in population-based search optimization methods, a high diversity is necessary during the early part of the search to allow using the full range of search space. On the other hand, during the latter part of the search, when the algorithm is converging nearby an optimal solution, fine-tuning algorithms are important to meet a global optima for effectiveness of finding a good UC planning solution. In our algorithm, the agents move effectively, the parameters are selected and adjusted adaptively. It has fewer restrictions on AS parameters presetting, requires less computational time, and has better clustering quality.

\subsection{Selective self-adaptive ant populations}

In our design, the hours of UC planning are petitioned into two groups that are "Least Path Hour (LPH)" and "Non-least Path Hour (NPH)". For the first group, the hours which give the highest pheromone trail intensity on the lowest economic dispatch cost among the candidate paths of CPMM, it can be set to the LPH(s). For the second group, the hours that give the highest pheromone on the other paths, but not the lowest economic dispatch cost can be set to the NPH(s). The ant populations then immigrate from LPH to NPH according to the proposed formula of Sharing Population Index (SPI) in (11). The SPI is proportional to the inverse of the difference of pheromone trail intensity on the NPH between the maximum value and the average value among the candidate path groups of CPMM. If there is much difference, we can know that it needs more ant populations to make a clear decision by pheromone trail information, and to reduce an ambiguous one by spreading the population to other candidate paths. By this way, the proposed SPI can be viewed as the alimentation of population distributing based on the remaining pheromone trail intensity left by ant agents. Where $t n$ is the index of NPH, $(t n=1,2, \ldots, T N) . T N$ is the number of all NPH(s). $\tau^{\max }{ }_{t n, u}$ and $\tau^{\text {mean }}{ }_{t n, u}$ are the maximum and the mean value of pheromone trail intensity among candidate paths of $t n \mathrm{NPH}$ at iteration $u$, respectively.

$$
\begin{aligned}
& S P I_{t n, u}=\frac{1 /\left[\tau_{t n, u}^{\max }-\tau_{t n, u}^{\text {mean }}\right]}{\sum_{t n=1}^{T N} 1 /\left[\tau_{t n, u}^{\max }-\tau_{t n, u}^{\text {mean }}\right]} \\
& I P I_{t n, u}=S P I_{t n, u} x P O P_{t l, u}
\end{aligned}
$$

After we can get the SPI(s) of all NPH(s), those SPI(s) are ranked; the highest SPI would be on the top rank. Then, the Immigrated Population Index (IPI) can be calculated as shown in (12), where $P O P_{t l, u}$ is the number of ant population in $t l \mathrm{LPH}$ at iteration $u$ by ranking of highest pheromone trail intensity contained in the candidate paths, $(t l=1,2, \ldots, T L) . T L$ is the number of all LPH(s). So, the $\mathrm{LPH}(\mathrm{s})$ which have high pheromone trail intensity would be raised 
to the upper rank, and get ready for emigrating ant agents to other $\mathrm{NPH}(\mathrm{s})$. The immigration can be maintained in such a way that the population of NPH(s) would be increased, and the population of $\mathrm{LPH}(\mathrm{s})$ would be decreased referring to the proposed SPI. By matching of both group's ranking with the same index, starting at $t l=1$ and $t n=1$, the immigration process is restricted to transfer only the number of populations that LPH(s) can support to NPH(s). Limitation of transferred ant agents is also presented here for the suitable number to maintain some ant agents on $\mathrm{LPH}(\mathrm{s})$ everlastingly. In this paper, we set the initial of ant populations $\left(P O P_{i n t}\right)$ for all hours as the round number of (13), the lower limit is set to the half of initial value, and the upper limit is set to the triple of initial value.

$$
P O P_{\text {int }}=N \exp \left(\frac{N}{T * 10}\right)
$$

When using this proposed concept, the ant population in each UC hour would not be equal; depending on whether the classification is LPH or NPH. The NPH(s) that ant agents flow into means those hours need more agents for better decision by the pheromone laying down process. Inversely, the LPH(s) that ant agents go out means those hours can have enough agents for good decision making. There is less chance that the solution will be stuck on a local optima because the classified hours can be changed in every iteration based on the probabilistically search concept. These behaviors give us a benefit that the presetting initial populations are of less significance for the proposed method. The SSAS provides automatically distribution for the number of ant populations to UC hours so that the fitness of each agent is greatly improved by this reasonable reinforcement.

\subsection{Selective self-adaptive transition probability parameters}

In this section, we also propose a new method to adjust transition probability parameters of trail decision formula in (14) adaptively. These parameters, represented as alpha $\left(\alpha_{t, u}\right)$ and beta $\left(\beta_{t, u}\right)$, control the relative importance of the pheromone trail and the visibility, respectively.

$$
p_{t, u}^{j}=\frac{\left[\tau_{t, u}^{j}\right]^{\alpha_{t, u}}\left[Q / F_{t, u}^{j}\right]^{\beta_{t, u}}}{\sum_{j=1}^{J_{t, u}}\left[\tau_{t, u}^{j}\right]^{\alpha_{t, u}}\left[Q / F_{t, u}^{j}\right]^{\beta_{t, u}}}
$$

Where $p_{t, u}^{j}$ is the transition probability of $j$ candidate path of $t$ hour at $u$ iteration, $\left(j=1,2, \ldots J_{t, u}\right), J_{t, u}$ is maximum candidate path of $t$ hour at $u$ iteration. $\tau_{t, u}^{j}$ is the pheromone intensity of $j$ candidate path of $t$ hour at $u$ iteration. $F_{t, u}^{j}$ is the fuel cost by economic dispatch of $j$ candidate path of $t$ hour at $u$ iteration. $Q$ is a constant related the quantity of trail laid by ants.

Setting $\alpha_{t, u}$ equal to zero, pheromone trail level is no longer considered, and a stochastic greedy algorithm would dominate. On the other hand, setting $\beta_{t, u}$ equal to zero, the visibility is to be ignored. Trading off between them ordering to the ant population in each UC hour is essential and significantly resulted to the convergence of UC solution. Conventionally, it always set to constant value at all times of searching [7]. So, this paper proposes a new time-varying adjustment of both parameters for suitable tuning between the visibility and pheromone trail level of each UC hour depending on the number of population via Gaussian function as shown in (15).

$$
g\left(x_{t, u}\right)=\exp \left(-x_{t, u}^{2}\right)
$$

$$
x_{t, u}=\frac{\left|P O P_{t, u}-P O P_{t, u-1}\right|}{P O P_{t, u-1}}
$$

Where $P O P_{t, u}$ is the number of the present ant population of hour $t$ at iteration $u, P O P_{t, u-l}$ is the number of the previous ant population of hour $t$ at iteration $u-1$. The property of Gaussian function gives a range between 1 to 0 , when $x_{t, u}$ in (16) is the ratio of the different population and the previous population of hour $t$ at iteration $u$. On the other hand, If Gaussian function is equal to 0 , it means $P O P_{t, u}$ is the same as $P O P_{t, u-l}$, and it would be more than 0 when it has population transferring. As shown in (17)-(20), in case of increasing population of UC hours, $\alpha_{t, u}$ is adapted in the direction to increase $(+)$ the importance of pheromone trail, and to decrease (-) $\beta_{t, u}$ concerning to the importance of visibility for better search decision of NPH.

$$
\begin{aligned}
& \text { For increasing population: } \\
& \qquad \begin{array}{c}
\alpha_{t, u}=\alpha_{t, u-1}+\beta_{\mathrm{int}} \beta_{t, u-1}\left(1-g\left(x_{t, u}\right)\right) \\
\beta_{t, u}=\beta_{t, u-1}-\alpha_{\mathrm{int}} \alpha_{t, u-1}\left(1-g\left(x_{t, u}\right)\right)
\end{array}
\end{aligned}
$$

For decreasing population:

$$
\begin{aligned}
& \alpha_{t, u}=\alpha_{t, u-1}-\frac{\beta_{\mathrm{int}} \beta_{t, u-1}\left(1-g\left(x_{t, u}\right)\right)}{\beta_{t, u-1}} \\
& \beta_{t, u}=\beta_{t, u-1}+\alpha_{\mathrm{int}} \alpha_{t, u-1}\left(1-g\left(x_{t, u}\right)\right)
\end{aligned}
$$

Oppositely, if decreasing population happens, $\alpha_{t, u}$ is adapted in the direction to decrease (-) the importance of pheromone trail, and to increase $(+)$ the importance of visibility for improving speed of searching, and it also tends to emigrate ant agents to help other UC hours in next iteration. For this proposed method in the second term of (17)-(20), we design these formulas in the fashion of swapping both parameters and multiplying them by the term of Gaussian function to reduce the significance of presetting initial value of $\alpha_{i n t}$ and $\beta_{\text {int }}$, and also to give the benefit of pulling back the step changing when the adjusting goes to wrong direction. Formally, the pheromone trail information still has more essential than the visibility for AS search guidance. As is shown in (19), we divide the second term by the bias parameter of $\beta_{t, u-I}$ to delay the decreasing of $\alpha_{t, u}$. When adaptive transition probability parameters are applied, the adjusting would result in the transition probability in (14). Too small $\alpha_{t, u}$ and large $\beta_{t, u}$ causes little chance of the diversity, while small $\beta_{t, u}$ and large $\alpha_{t, u}$ gives strong concern of the pheromone trail importance more than the visibility when SSAS needs to reinforce decision process of a local search, and to diverge UC solution far away form a local optima. In this paper, we set the minimum and maximum range of 1 to 5 for both $\alpha_{t, u}$ and $\beta_{t, u}$.

\subsection{Updating pheromone based diversity control}

The number of ant populations are performed with respect to above the given procedures, whereas the pheromone updating strategy is made probabilistically based on the diversity control approach [12] in the following equations:

$$
\tau_{t, u}^{j}=\rho \tau_{t, u-1}^{j}+\left(\Delta \tau_{t, u}^{j}+\Delta \tau_{t, u}^{b e s t}\right)
$$




$$
\begin{aligned}
\Delta \tau_{t, u}^{j} & =\sum_{k=1}^{K_{t, u}}\left(K_{t, u}-k\right)\left(Q / F_{t, u}^{j}\right) \\
\Delta \tau_{t, u}^{b e s t} & = \begin{cases}K_{t, u}\left(Q / F T_{u}^{b e s t}\right) & \text { Best path } \\
0 & \text { Non-best path }\end{cases}
\end{aligned}
$$

Where $k$ is the index of ant agent $\left(k=1,2, \ldots . K_{t, u}\right), K_{t, u}$ is the maximum number of ant agents of $t$ hour at $u$ iteration, $F T^{\text {best }}{ }_{u}$ is the best (lowest) total operating cost of $u$ iteration. $\rho$ is the constant of pheromone evaporation, in this paper we set $\rho=0.5$ and $Q=10000$. By these rules, pheromone intensity will be updated based the diversity control to favor the exploration of different paths during the whole search process. Therefore, the desirability of candidate paths can be dynamically changed. Moreover, transition probability parameters can be adaptively related to the concept of the proposed selective selfadaptive approach. It allows agents to find better paths so far using pheromone information based adaptive populations. The optimal solution can be enhanced through these processes.

\section{SIMULATION RESULTS}

The objective of this section is to illustrate the capability of the proposed algorithm in terms of SSAS solutions quality and CPU time requirements. The original system consisted of 10 generating units [13] whose load demand over a planning horizon of one day, was divided into 24 hours and generating parameters are provided in the Appendix (see Table A and B). The 20, 40, 60, 80 and 100 units data are obtained by replicating the base case (10 units), whereas the load demands are adjusted in proportion to the system sizes. In the simulation, the spinning reserve requirement in each hour is assumed to be $10 \%$ of the power demand during that hour without violating any ramp rate constraints.

The UC simulation results of the proposed SSAS are compared against several UC solution methods taken from the previous publications in the following: Lagrangian Relaxation (LR) [13],
Genetic Algorithms [13], Evolutionary Programming (EP) [6], Improved Cooperative Genetic Algorithms (ICGA) [14], Genetic Algorithms for Unit Commitment (GAUC) [15], Fast Genetic Algorithms (FGA) [16], and Enhanced Lagrangian Relaxation (ELR) [17]. For confirming the performance, the proposed SSAS is also compared to the results of AS based the diversity control approach (DAS) [9]. All computations for the proposed SSAS method, and DAS as well, are performed on Pentium M, 1.5 MHz based M-file source codes of MATLAB programming.

The comparison results of the total operating costs with the literature results are shown in Table 1. As shown, the costs obtained from the SSAS approach are equal to some methods in the case of 10 and 20 units systems, but significantly lower than those from the literature methods for different numbers of 40 up to 100 units.

It is also shown that because of a good sharing in ant populations based the selective self-adaptive approach, so that SSAS is found to be the best economical saving solution over the DAS. Table 2 provides a CPU times comparison of SSAS with the previous literature. From the aspect of computational efficiency, although they may not be directly comparable with the previous literatures due to different computers used, the trend of computational times of 10 up to 100 -unit system shown that SSAS slightly provides a reasonable computational time in every unit cases. In cases of 10 up to 20 units, although SSAS takes a little longer than that by the ELR, but in cases of the large-scale UC problem of 40 up to 100-unit, SSAS can reach faster CPU time accompany with much lower total operating UC cost than those by the literature methods. Moreover, with the concept of proposed SSAS, the CPU time acquired by SSAS is also remarkably lower than DAS.

To demonstrate the changing of ant populations in each hour of UC planning applied with the proposed SSAS, Fig. 4 shows the population adaptations to reach the solution of 80-unit test system. All UC hours are assigned by the initial value of ant population at 112 ant populations, that is determined by (13) according to the number of generating units in 24 hours.

\begin{tabular}{|c|c|c|c|c|c|c|c|c|c|}
\hline \multirow[t]{2}{*}{ Units } & \multicolumn{8}{|c|}{ Total Operating Costs $(\$)$} & \multirow[b]{2}{*}{ SSAS } \\
\hline & $\begin{array}{c}L R \\
{[13]}\end{array}$ & $\begin{array}{l}G A \\
{[13]}\end{array}$ & $\begin{array}{l}E P \\
{[6]}\end{array}$ & $\begin{array}{c}C G A \\
{[14]}\end{array}$ & $\begin{array}{c}G A U C \\
{[15]}\end{array}$ & $\begin{array}{l}F G A \\
{[16]}\end{array}$ & $\begin{array}{l}E L R \\
{[17]}\end{array}$ & $\begin{array}{c}D A S \\
{[9]}\end{array}$ & \\
\hline 10 & 565,825 & 565,825 & 564,551 & 566,404 & 563,977 & - & 563,977 & 563,977 & 563,977 \\
\hline 20 & $1,130,660$ & $1,126,243$ & $1,125,494$ & $1,127,244$ & $1,123,516$ & - & $1,123,297$ & $1,125,826$ & $1,123,297$ \\
\hline 40 & $2,258,503$ & $2,251,911$ & $2,249,093$ & $2,254,123$ & $2,249,715$ & $2,247,336$ & $2,244,237$ & $2,254,094$ & $2,243,588$ \\
\hline 60 & $3,394,066$ & $3,376,625$ & $3,371,611$ & $3,366,210$ & $3,375,065$ & $3,367,637$ & $3,363,491$ & $3,374,039$ & $3,362,824$ \\
\hline 80 & $4,526,022$ & $4,504,933$ & $4,498,479$ & $4,498,943$ & $4,505,614$ & $4,491,509$ & $4,485,633$ & $4,495,730$ & $4,484,591$ \\
\hline 100 & $5,657,277$ & $5,627,437$ & $5,623,885$ & $5,630,838$ & $5,626,514$ & $5,610,855$ & $5,605,678$ & $5,627,847$ & $5,603,186$ \\
\hline
\end{tabular}

Table 1. The comparison results of $\mathrm{UC}$ solution

\begin{tabular}{|c|c|c|c|c|c|c|c|c|c|}
\hline \multirow[t]{3}{*}{ Units } & \multicolumn{9}{|c|}{ CPU Time (s) } \\
\hline & $L R$ & $G A$ & $E P$ & $I C G A$ & $G A U C$ & $F G A$ & $E L R$ & $D A S$ & $S S A S$ \\
\hline & [13] & [13] & [6] & [14] & [15] & [16] & [17] & [9] & \\
\hline 10 & - & 221 & 100 & - & 85 & - & 4.0 & 157 & 8.0 \\
\hline 20 & - & 733 & 340 & - & 225 & - & 16 & 352 & 18 \\
\hline 40 & - & 2697 & 1176 & - & 614 & 29 & 52 & 749 & 27 \\
\hline 60 & - & 5840 & 2267 & - & 1085 & 42 & 113 & 1240 & 38 \\
\hline 80 & - & 10036 & 3584 & - & 1975 & 60 & 209 & 2508 & 43 \\
\hline 100 & - & 15733 & 6120 & - & 3547 & 75 & 345 & 4965 & 62 \\
\hline
\end{tabular}

Table 2. The comparison results of CPU time 


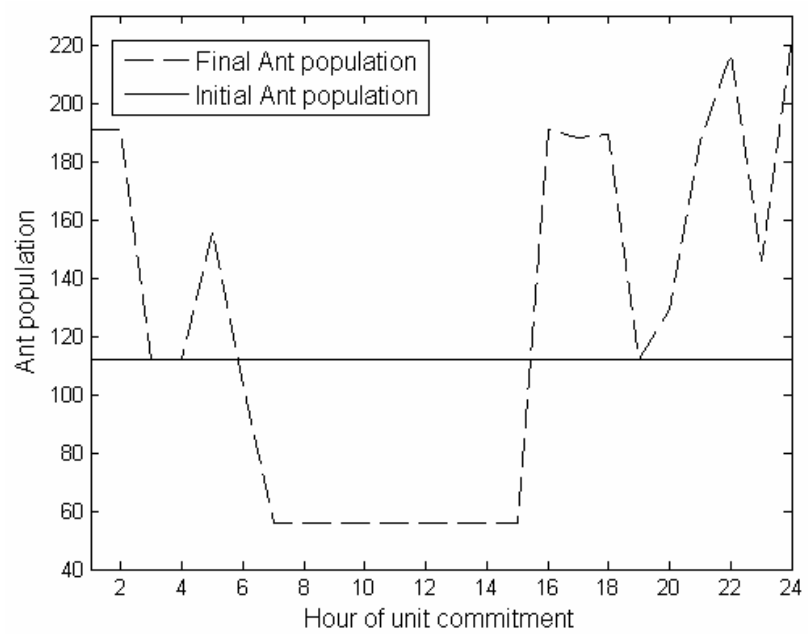

Figure 4. Populations adaptation of 80-unit system

As shown in Fig 4., SSAS decreases ant populations in the hours that the cheapest economic dispatch cost can give the highest pheromone paths of CPMM, which means it can provided a good decision by itself so that emigrating ant populations to help other UC hours would probably be better, and it also helps to reduce overall CPU time of searching. The flat dash line between the UC hours 7 to 15 is the result of ant populations maintaining to reserve the agents being higher than the lower limit of (13).

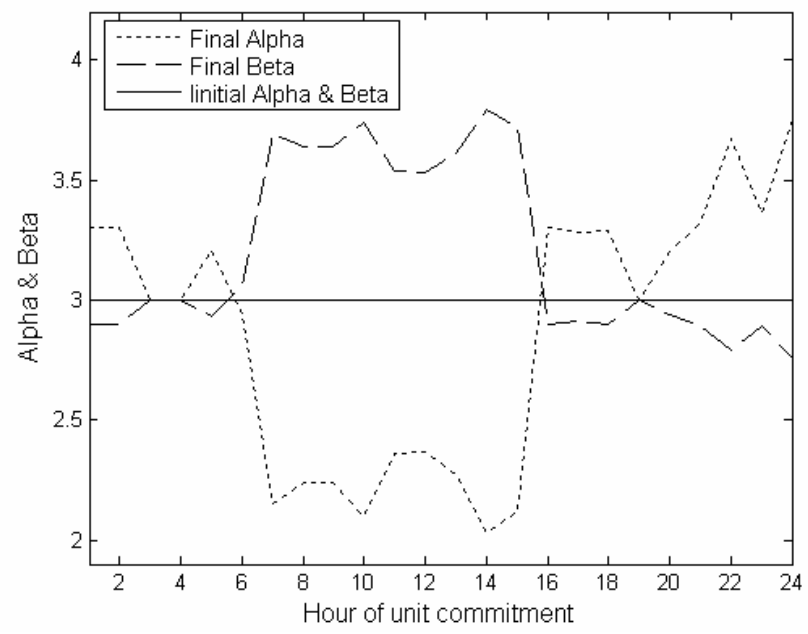

Figure 5. Alpha and Beta adaptation of 80-unit system

For adaptation result in transition probability parameters of $\alpha_{t, u}$ and $\beta_{t, u}$, Fig. 5 shows the changing of $\alpha_{t, u}$ and $\beta_{t, u}$. It has been shown that there is a significant relation between the transition probability parameters and the number of ant population in each UC hour. Respecting to (17)-(20), when the number of ant populations are increased, $\alpha_{t, u}(\mathrm{~s})$ are increased and $\beta_{t, u}(\mathrm{~s})$ are decreased concerning the pheromone trail information which comes from the transferred agents. Conversely, when SSAS decreases populations, $\alpha_{t, u}$ (s) are decreased and $\beta_{t, u}$ (s) are increased to accelerate the search process concerning the economic dispatch cost results more than pheromone trail information provided by ant agents. Fig. 6 shows the comparison of solution convergence trends of 80-unit system between DAS and SSAS. Ant system algorithm is a stochastic technique. In order to test the robustness of the proposed method, we made the competition of
15 iterations searching. Each algorithm was run five times and the results shown in this figure are averages across those five runs of its best solution. It is seen that SSAS has noticeably improved the speed of convergence. It is clearly better when compared to DAS.

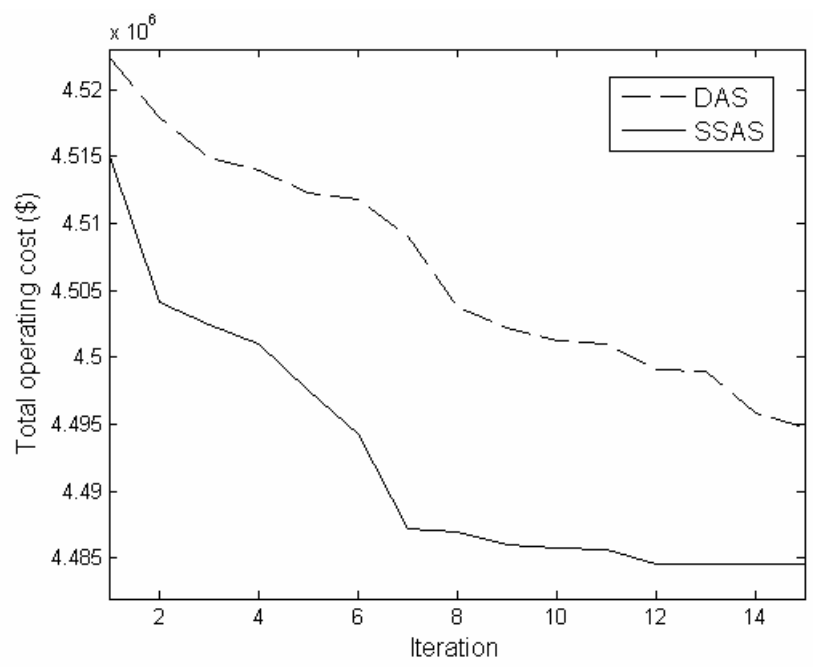

Figure 6. UC solution convergence of 80-unit system

Simulation results shown that SSAS scheme can effectively improve UC solution optimality at a sight cost of less computational time when compared to the literatures. These potentials and effectiveness of the proposed SSAS show that it has an outstanding performance, which is favorable for a large-scale constrained UC problem implementation.

\section{CONCLUSIONS}

A new algorithm of ant system based on the selective self-adaptive approach has been presented to the constrained unit commitment problem. It is necessary to enhance a standard AS performance based UC problem with the addition of an adaptive algorithms for the number of ant populations and transition probability parameters in order to obtain a satisfactory UC solution within a reasonable CPU time. By avoiding the infeasible of UC solutions and recovering highquality candidate path neighbors, the effective repairing heuristic module incorporated with the candidate path management module is introduced as an effective tool for UC problem solution so that the execution time of the proposed SSAS is significantly reduced. The performance of the proposed SSAS algorithm is compared to those of the existing literature methods in dealing with the constraints of UC problem and reaching better UC solutions have been validated in this paper. Additionally, due to the proposed SSAS affords the benefits of good adaptability and reasonability to operate based on the population adjustments and the pheromone trail intensity evaluations, simulation results in scheduling of a test system up to 100 units is shown that the searching convergence of SSAS is noticeably improved when compared to the DAS. These advantages indicate that the proposed SSAS has satisfactory performance for applying to unit commitment problems.

\section{REFERENCES}

[1] A.J. wood and B. F. Wollenberg, Power Generation Operation and Control, $2^{\text {nd }}$ ed., New York: John Wiley \&Sons, Inc., 1996.

[2] G.B. Sheble, G.N. Fahd, "Unit commitment literature synopsis," IEEE Trans. on Power Systems, vol. 9, no. 1, pp. 128-135, 1994.

[3] F. Zhuang and F. D. Galiana, "Towards a more rigorous and practical unit commitment by Lagrangian relaxation," IEEE Trans. on Power Systems, vol. 3, no. 2, pp. 763-772, 1988 
[4] S. Virmani, E. C. Adrian, K. Imhof and S. Mukherjee, "Implementation of a Lagrangian relaxation based unit commitment problem," IEEE Trans. on Power Systems, vol. 4, no. 4, pp. 1373$1380,1989$.

[5] T. T. Maifeld and G. B. Sheble, "Genetic-based unit commitment algorithm," IEEE Trans. on Power Systems, vol. 11, no. 3, pp. 13591372, 1996.

[6] K. A. Juste, H. Kita, E. Tanaka, and J. Hasegawa, "An evolutionary programming solution to the unit commitment problem," IEEE Trans. on Power Systems, vol. 14, pp. 1452-1459, Nov. 1999.

[7] M. Dorigo, V. Maniezzo and A. Colorni, "Ant system: optimization by a colony of cooperating agents," IEEE Trans. on System, Man and Cybernetics, Part B, vol. 26, no. 1, pp. 29-41, 1996.

[8] S.J. Huang, "Enhancement of hydroelectric generation scheduling using ant colony system based optimization approaches," IEEE Trans. on Energy Conversion, vol. 16, pp. 296-301, Sept. 2001.

[9] D. Nualhong, S. Chusanapiputt, T. Saengsuwan, S. Phoomvuthisarn and S. Jantarang "Diversity control approach to ant colony optimization for unit commitment problem," in Proc. International Technical Conference of IEEE Region 10, November 2004.

[10] M. Dorigo, "Optimization, learning, and natural algorithms," Ph.D. dissertation (in Italian), Dipartimento di Elettronica, Politecnico di Milano, Milano, Italy, 1992.

[11] M. Dorigo and L.M. Gambardella, "Ant colony system: a cooperative learning approach to the traveling salesman problem," IEEE Trans. on Evol. Compiut., vol. 1, pp 53-66, Apr. 1997.

\section{Appendix}

Table A: Load demand

\begin{tabular}{c|c|c|c|c|c}
\hline Hour & Load (MW) & Hour & Load (MW) & Hour & Load (MW) \\
\hline 1 & 700 & 9 & 1300 & 17 & 1000 \\
\hline 2 & 750 & 10 & 1400 & 18 & 1100 \\
\hline 3 & 850 & 11 & 1450 & 19 & 1200 \\
\hline 4 & 950 & 12 & 1500 & 20 & 1400 \\
\hline 5 & 1000 & 13 & 1400 & 21 & 1300 \\
\hline 6 & 1100 & 14 & 1300 & 22 & 1100 \\
\hline 7 & 1150 & 15 & 1200 & 23 & 900 \\
\hline 8 & 1200 & 16 & 1050 & 24 & 800 \\
\hline
\end{tabular}

Table B: Unit data for the ten-unit system

\begin{tabular}{lccccc}
\hline & Unit1 & Unit2 & Unit3 & Unit4 & Unit5 \\
\hline Pmax $(\mathrm{MW})$ & 455 & 455 & 130 & 130 & 162 \\
\hline Pmin(MW) & 150 & 150 & 20 & 20 & 25 \\
\hline $\mathrm{c}(\$ / \mathrm{h})$ & 1000 & 970 & 700 & 680 & 450 \\
\hline $\mathrm{b}(\$ / \mathrm{MWh})$ & 16.19 & 17.26 & 16.60 & 16.50 & 19.70 \\
\hline $\mathrm{a}\left(\$ / \mathrm{MW}^{2}-\mathrm{h}\right)$ & 0.00048 & 0.00031 & 0.002 & 0.00211 & 0.00398 \\
\hline Min up $(\mathrm{h})$ & 8 & 8 & 5 & 5 & 6 \\
\hline Min down $(\mathrm{h})$ & 8 & 8 & 5 & 5 & 6 \\
\hline Hot start cost $(\$)$ & 4500 & 5000 & 550 & 560 & 900 \\
\hline Cold start cost $(\$)$ & 9000 & 10000 & 1100 & 1120 & 1800 \\
\hline Cold start hour $(\mathrm{h})$ & 5 & 5 & 4 & 4 & 4 \\
\hline Initial status $(\mathrm{h})$ & 8 & 8 & -5 & -5 & -6 \\
\hline & & & & & \\
\hline & Unit6 & Unit7 & Unit8 & Unit9 & Unit10 \\
\hline Pmax $(\mathrm{MW})$ & 80 & 85 & 55 & 55 & 55 \\
\hline Pmin(MW) & 20 & 25 & 10 & 10 & 10 \\
\hline $\mathrm{c}(\$ / \mathrm{h})$ & 370 & 480 & 660 & 665 & 670 \\
\hline $\mathrm{b}(\$ / \mathrm{MWh})$ & 22.26 & 27.74 & 25.92 & 27.27 & 27.79 \\
\hline $\mathrm{a}\left(\$ / \mathrm{MW}{ }^{2}-\mathrm{h}\right)$ & 0.00712 & 0.00079 & 0.00413 & 0.00222 & 0.00173 \\
\hline Min up $(\mathrm{h})$ & 3 & 3 & 1 & 1 & 1 \\
\hline Min down $(\mathrm{h})$ & 3 & 3 & 1 & 1 & 1 \\
\hline Hot start cost $(\$)$ & 170 & 260 & 30 & 30 & 30 \\
\hline Cold start cost $(\$)$ & 340 & 520 & 60 & 60 & 60 \\
\hline Cold start hour $(\mathrm{h})$ & 2 & 2 & 0 & 0 & 0 \\
\hline Initial status $(\mathrm{h})$ & -3 & -3 & -1 & -1 & -1 \\
\hline & & & & & \\
\hline
\end{tabular}

[12] B. Bullnheimer, R. F. Hartl and C. Strauss, "A new rank based version of the ant system: a computational study," Central European Journal for Operations Research and Economics, vol. 17, no. 1, pp. 25-38, 1999.

[13] S.A. Kazarlis, A. G. Bakirtzis, and V. Petridis, "A genetic algorithm solution to the unit commitment problem," IEEE Trans. on Power Systems, vol. 11, no. 1, 1996, pp 83-92.

[14] I. G. Damousis, A. G. Bakirtzis, and P. S. Dokopoulos, "A solution to the unit-Commitment problem using integer-coded genetic algorithm," IEEE Trans. on Power Systems, vol. 19, no.2, pp.1165-1172, May. 2004.

[15] T. Senjyu, H. Yamashiro, K. Uezato, and T. Funabashi, "A unit commitment problem by using genetic algorithms based on characteristic classification," in Proc. IEEE/Power Eng. Soc. Winter Meeting, vol. 1, 2002, pp. 58-63.

[16] T. Senjyu, H. Yamashiro, K. Shimabukuro, K. Uezato, T. Funabashi, "Fast solution technique for large-scale unit commitment problem using genetic algorithm," IEE Proc.-Gener. Transm. Distrib., vol. 150, no. 6 , pp 753-760, Nov. 2003.

[17] W. Ongsakul, and N. Petcharaks, "Unit commitment by enhanced adaptive Lagrangian relaxation," IEEE Trans. on Power Systems, vol. 19, no.1, pp.620-628, Feb. 2004.

Songsak Chusanapiputt is a charter engineer of Thailand. He received the B.Eng. in Electrical Engineering from Kasetsart University, Thailand in 1983, the M.Eng. and D.Eng. degree in Electrical Engineering from Chulalongkorn University, Thailand in 1985 and 1991, respectively. He is presently a lecturer in the Department of Electrical Power Engineering, Mahanakorn University of Technology.

Dulyatat Nualhong received the B.Eng. degree in Electrical Enginering from Suranaree University of Technology, Thailand and the M.Eng. degree in Electrical Engineering form Mahanakorn University of Technology, Thailand in 1999 and 2002, respectively. Currently, he is an electrical engineer with the Electrical Engineer Department, Electrical and Control System Engineering Division, Electricity Generating Authority of Thailand (EGAT). He is now pursuing the D.Eng. degree in Electrical Engineering at Mahanakorn University of Technology.

Sujate Jantarang is an Associate Professor in the Department of Computer Engineering, Mahanakorn University of Technology, Thailand. He received the B. Eng. and D. Eng. degree in Electrical Engineering from King Mongkut's Institute of Technology Ladkrabang (KMITL), Thailand in 1984 and 1990, respectively. He is now the director of Satellite Research Center, and the Vice President of Mahanakorn University of Technology.

Sukumvit Phoomvuthisarn is a charter engineer of Thailand. He received the B. Eng. degree in Electrical Engineering from Chulalongkorn University, Thailand in 1971, the M.S. and Ph. D. degree in Electrical Engineering from University of Cincinnati, Cincinnati, Ohio, in 1973 and 1975 respectively. Since 1975, he has been with the Department of Electrical Engineering, Chulalongkorn University, Thailand, where he is currently an Associate Professor. 